\title{
Three-dimensional simulation of crack propagation in ferroelectric polycrystals: effect of combined toughening mechanisms
}

\author{
Amir Abdollahi, Irene Arias \\ Laboratori de Càlcul Numèric (LaCàN), Departament de Matemàtica Aplicada III, \\ Universitat Politècnica de Catalunya (UPC), Campus Nord UPC-C2, E-08034 \\ Barcelona, Spain
}

\begin{abstract}
We simulate the fracture processes of ferroelectric polycrystals in three dimensions using a phase-field model. In this model, the grain boundaries, cracks and ferroelectric domain walls are represented in a diffuse way by three phase-fields. We avoid thereby the difficulty of tracking the interfaces in three dimensions. The resulting model can capture complex interactions between the crack and the polycrystalline and ferroelectric domain microstructures. The simulation results show the effect of the microstructures on the fracture response of the material. Crack deflection, crack bridging, crack branching and ferroelastic domain switching are observed to act as the main fracture toughening mechanisms in ferroelectric polycrystals. Our fully 3-D simulations illustrate how the combination of these mechanisms enhances the fracture toughness of the material, and pave the way for further systematic studies, including fracture homogenization.

Keywords: Polycrystals, Ferroelectricity, Fracture, Phase-field models, Finite element analysis
\end{abstract}




\section{Introduction}

Ferroelectric materials are important to a broad range of applications ranging from common rail fuel injection, to medical ultrasound, structural health monitoring and prognosis, and various types of accelerometer and vibrational gyro sensors. However, the reliability of these systems is an important concern due to the inherent brittleness of ferroelectric ceramics, demanding investigations on their complex fracture behavior (see $[1,2,3]$ for comprehensive reviews of the recent works). Complexity stems mainly from the interactions between the cracks, material microstructure (both ferroelectric domains and polycrystalline grains) and localized stress and electric fields
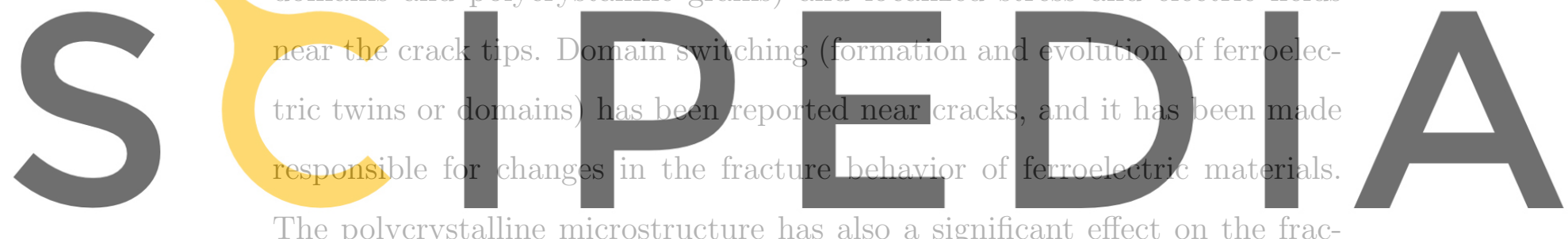

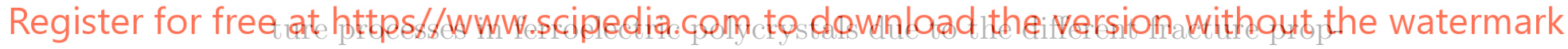

erties of the grain boundaries and the bulk. Therefore, these microstructure

effects should be taken into account in the analysis of the global reliability of ferroelectric components.

A number of theoretical approaches have been developed to understand fracture phenomena in ferroelectric ceramics. These include the models based on the linear theory of piezoelectricity, where microstructure effects are not taken into account $[4,5,6,7]$. Such approaches have allowed researchers to study the basic concepts of the linear theory in the context of fracture mechanics. To account for the domain microstructure, three sets of models have been developed, which are (1) phenomenological models describing im- 
plicitly the domain formation around the cracks [8, 9], (2) models relying on an energy-based switching criterion [10], considering the local phase transformations near the crack tip under the assumption of small-scale switching $[11,12,13,14]$, and (3) phase-field or time-dependent Devonshire-GinzburgLandau (TDGL) models describing explicitly the formation and evolution of individual ferroclectric domains around the cracks $[15,16,17,18,19]$. Related models have also taken into account the effects of polycrystalline microstructure, particularly the grain orientations [20, 21].

The above mentioned models of ferroelectric fracture consider fixed or stationary crack configurations. Recently, a cohesive zone finite element model


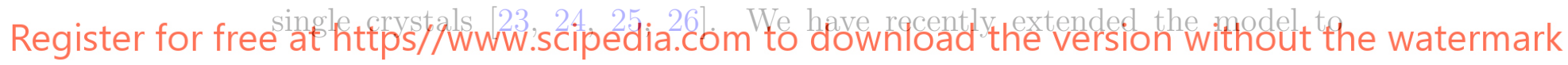

ferroelectric polycrystals by introducing polycrystalline microstructures [27].

With this model we have shown the intergranular and transgranular modes of fracture and the interactions of the microstructures and the crack. These interactions lead to crack deflection and ferroelastic domain switching as the toughening mechanisms in ferroelectric polycrystals observed in experiment [28]. Although the 2-D simulations in [27] were able to capture qualitatively some of the toughening mechanisms, the fracture response of ferroelectric polycrystals is intrinsically three-dimensional. The main objective of this paper is to extend the model to three dimensions and to produce more realistic simulation results in 3-D, explaining the complex fracture processes and 
toughening mechanisms in these materials. In particular, we aim to evaluate the combination of the toughening mechanisms for enhancing the fracture toughness of the material.

The structure of the paper is as follows. The theory of the extended model is presented in Section 2. Numerical simulations are presented in Section 3, along with a discussion of the observed crack patterns and fracture mechanisms. The last section is the conclusion of this paper.

\section{Theory}

\section{This section presents the phase-field model of fracture in ferroelectric}
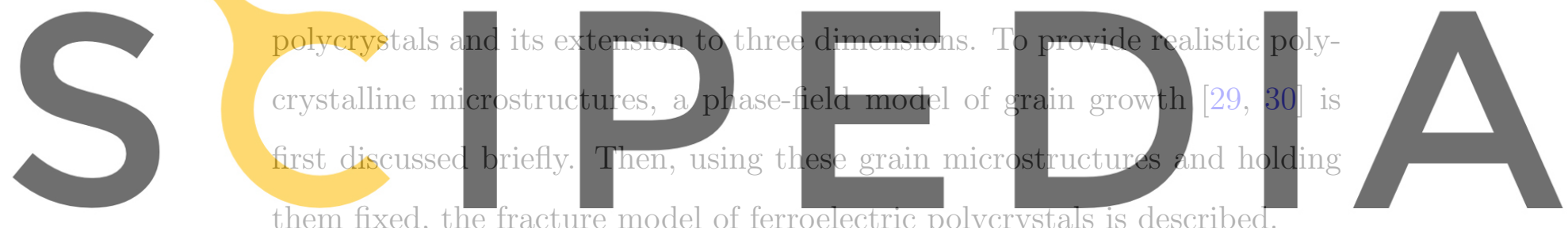

\section{Register for free at https//www.scipedia.com to download the version without the watermark \\ 2.1. Phase-field model for grain growth}

According to the phase-field model of grain growth $[29,30]$, the total free energy of a heterogeneous system is

$$
F=\int_{\Omega}\left[f_{0}\left(\eta_{1}, \eta_{2}, \ldots, \eta_{m}\right)+\sum_{i=1}^{m} \frac{\kappa_{i}}{2}\left(\nabla \eta_{i}\right)^{2}\right] \mathrm{d} \Omega,
$$

where $f_{0}$ is the local free energy density associated with the orientation field variables $\left(\eta_{1}, \eta_{2}, \ldots, \eta_{m}\right)$ for distinguishing different orientations of grains and $m$ is the number of possible orientations. $\kappa_{i}$ are the coefficients of the gradient energy terms penalizing sharp variations in the field variables. The free 
energy density $f_{0}$ is written as [29]

$$
\begin{aligned}
f_{0}\left(\eta_{1}, \eta_{2}, \ldots, \eta_{m}\right)= & \sum_{i=1}^{m}\left(-\frac{\alpha}{2} \eta_{i}^{2}+\frac{\beta}{4} \eta_{i}^{4}\right) \\
& +\gamma \sum_{i=1}^{m} \sum_{j \neq i}^{m} \eta_{i}^{2} \eta_{j}^{2},
\end{aligned}
$$

where $\alpha, \beta$ and $\gamma$ are positive constants characterizing the energy landscape.

For $\gamma>\beta / 2, f_{0}$ describes a multi-well energy landscape with $2 m$ minima located at $\left(\eta_{1}, \eta_{2}, \ldots, \eta_{m}\right)=(1,0, \ldots, 0),(0,1, \ldots, 0), \ldots,(0,0, \ldots$
$1),(-1,0$
$0),(0,-1$,
$0)$,
$(0,0, \ldots,-1)$. Each of these $2 m$

minima corresponds to a subset of all the grains in the polycrystal. The grain
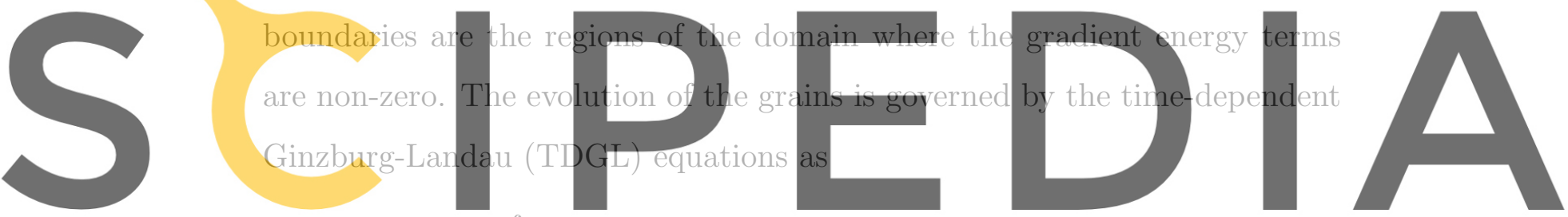

Register for free at https//ww-w.scipedia.com to download the version without the watermark

$$
\begin{aligned}
= & \int_{\Omega}\left(\alpha \eta_{i}-\beta \eta_{i}^{3}-2 \gamma \eta_{i} \sum_{j \neq i}^{m} \eta_{j}^{2}\right) \delta \eta_{i} \mathrm{~d} \Omega \\
& +\int_{\Omega}\left(\kappa_{i} \nabla^{2} \eta_{i}\right) \delta \eta_{i} \mathrm{~d} \Omega
\end{aligned}
$$

where $i=1,2, \ldots, m$ and $1 / \mu_{g}$ is the mobility of the process.

\subsection{Phase-field model for fracture of ferroelectric polycrystals}

The total electromechanical enthalpy of a possibly fractured ferroelectric polycrystal occupying a region $\Omega$ is written in terms of the mechanical displacement $\mathbf{u}$, the polarization $\mathbf{p}$, the electric potential $\phi$ and the phase-field $v$, as $[27]$ 


$$
\begin{aligned}
H[\mathbf{u}, v, \mathbf{p}, \phi]= & \int_{\Omega} h^{\text {poly }}(\varepsilon(\mathbf{u}), \mathbf{p}, \mathbf{E}(\phi), v) \mathrm{d} \Omega \\
& +G_{c}^{\text {poly }} \int_{\Omega}\left[\frac{(1-v)^{2}}{4 \kappa}+\kappa|\nabla v|^{2}\right] \mathrm{d} \Omega .
\end{aligned}
$$

where body loads, volume charges, tractions and surface charges have been ignored for simplicity. The first integral is referred to as total bulk energy of the material while the second integral takes the role of the surface energy.

The scalar field $v$ is the phase-field parameter describing a smooth transition in space between unbroken $(v=1)$ and broken $(v=0)$ states of the material.
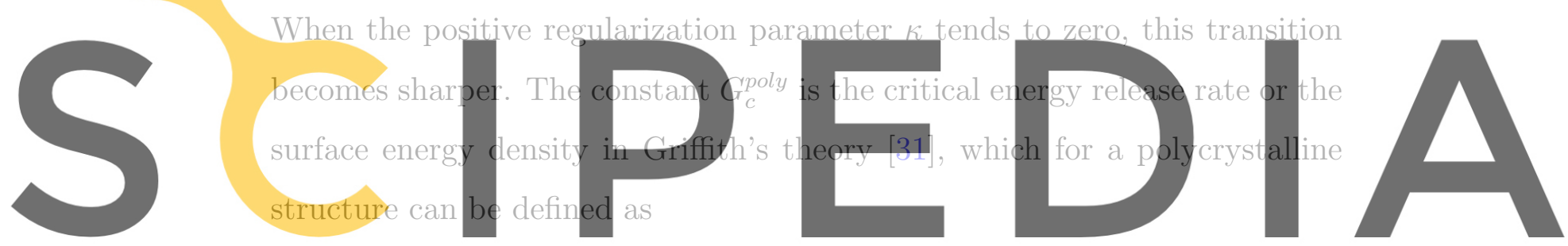

Register for free at https//www.scipedia. $\varepsilon_{c} m_{c}$ to downjoad the version without the watermark

where $G_{c}$ is the critical energy release rate of the bulk crystal, and the function $\mathscr{F}$ controls the weakening of the material at the grain boundaries. These boundaries can be identified through the function $\xi$ defined as

$$
\xi=\sum_{i=1}^{m} \eta_{i}^{2 k}
$$

This function has a unit value inside the grains and smaller positive values at the grain boundaries [29]. The function $\mathscr{F}$ attains its maximum for $\xi=1$ (inside the grains) and its minimum indicates the ratio of the critical fracture energy of the grain boundary to that of the grain interior. The parameter $k$ 
is a positive integer to control this ratio. The choice of the function $\mathscr{F}$ and parameter $k$ is discussed in Section 3.

The electro-mechanical enthalpy density $h^{\text {poly }}$ is given as

$$
h^{p o l y}(\varepsilon, \mathbf{p}, \mathbf{E}, v)=W_{e}(\varepsilon, v)+W_{f}(\varepsilon, \mathbf{p}, \mathbf{E}, v)
$$

where $W_{e}$ is the part of the bulk energy density associated with the strain and $W_{f}$ is the electromechanical energy density associated with the ferroelectric response. The energy density $W_{e}$ is written as


Register for free at https//www.scipedia.com to download the version without the watermark

components of the strain tensor. This decomposition is introduced to distin-

guish the contributions to the strain energy due to compression, expansion, and shear. As further discussed in the Conclusions, in the present setting this model distinguishes nominal compression or expansion relative to a reference cubic state. To prevent non-physical crack interpenetration in compressed regions, the compression term is not multiplied by the jump set function $\left(v^{2}+\eta_{\kappa}\right)[32]$.

By noting that $v^{2}$ multiplies the expansion and shear terms in Eq. (8), it is clear that $v=0$ reduces the stiffness of the material to zero in the fractured zone, leading to the full degradation of the elasticity in this zone. As a consequence, the numerical discretization method results in an ill-conditioned 
stiffness matrix. The parameter $\eta_{\kappa}$ is a small (relative to $\kappa$ ) residual stiffness to avoid this problem and to control the condition number of the matrix. This parameter must be chosen as small as possible in order not to add large artificial stiffness to the fractured zone.

The electromechanical energy density $W_{f}$ associated with polarization $\mathbf{p}$, electric field $\mathbf{E}, \varepsilon$ and $v$ is given as



where $\varepsilon_{0}$ is the permittivity of free space, $U$ is the domain wall energy density
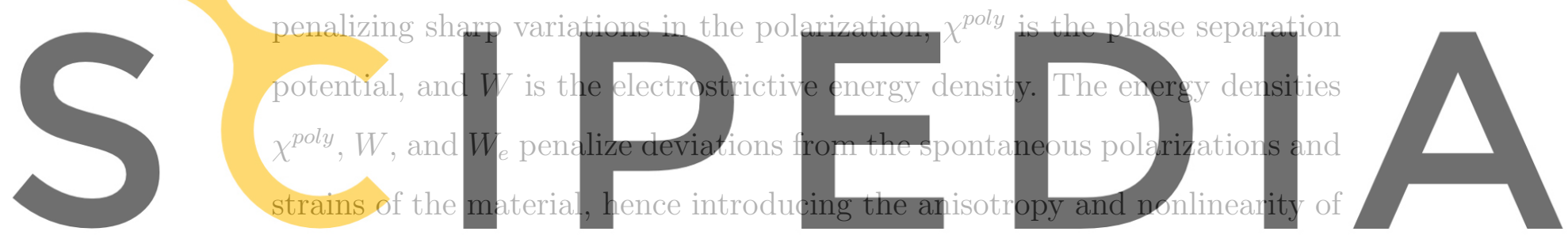

ferroelectric materials. Note that we assume the parent cubic paraelectric Register for free at https//www.scipedia.com to download the version without the watermark phase to be the reference configuration for the tension-compression decom-

position. Therefore, the spontaneous stress-free state is considered as a pre-

loaded state due to the structural changes during the phase transition or poling of the material. However, this state will not be damaged since the electrostrictive energy $W$ accommodates the structural changes and cancels out the induced elastic energy in Eq. (8).

The electromechanical enthalpy density $h^{\text {poly }}$ is obtained by transforming the energy terms of the ferroelectric single crystal from the local coordinate system of each individual grain to the global coordinate system of the polycrystal [27]. It can be shown that the energy density $W_{e}$, the domain wall energy density $U$, the electrostrictive energy density $W$ and the 
last two terms in Eq. (9) remain unchanged by this transformation. The only modified energy term is the phase-separation potential $\chi^{\text {poly }}$ obtained as $\operatorname{chi} i^{\text {poly }}(\mathbf{p})=\chi\left(\mathbf{p}^{L}\right)$, where $\chi$ and $\mathbf{p}^{L}$ are the phase-separation potential and the polarization, respectively, in the local coordinate system. The detailed formulation of the functions $U, W$, and $\chi$ in three dimensions is given in Appendix $A$.

We describe the orientation of each grain in the 3-D polycrystalline structure using three Euler angles $(\Phi, \Theta, \Psi)$, corresponding to three consecutive counter-clockwise rotations with respect to the global coordinates $\left(x_{1}, x_{2}, x_{3}\right)$ : $\Phi$ about the $x_{3}$-axis, $\Theta$ about the rotated $x_{1}^{\prime}$-axis and $\Psi$ about the newest


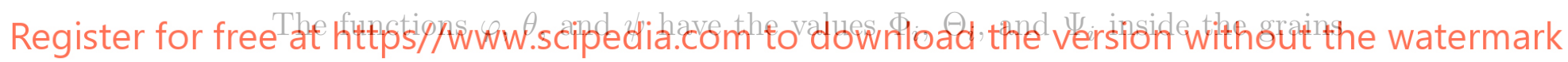

corresponding to the orientation field variable $\eta_{i}$. At the grain boundaries,

the numerators in Eq. (10) decrease significantly. The denominators compensate for this effect and assure a smooth transition between the orientations of adjacent grains across their boundaries. Then, the transformation matrix from the global to local coordinate system is given by

$\mathbf{T}=\left(\begin{array}{ccc}\cos [\varphi] \cos [\psi]-\cos [\theta] \sin [\varphi] \sin [\psi] & \sin [\varphi] \cos [\psi]+\cos [\theta] \cos [\varphi] \sin [\psi] & \sin [\theta] \sin [\psi] \\ -\cos [\theta] \cos [\psi] \sin [\varphi]-\cos [\varphi] \sin [\psi] & \cos [\theta] \cos [\varphi] \cos [\psi]-\sin [\varphi] \sin [\psi] & \sin [\theta] \cos [\psi] \\ \sin [\theta] \sin [\varphi] & -\cos [\varphi] \sin [\theta] & \cos [\theta]\end{array}\right)$ 
The polarization in the local coordinate system, $\mathbf{p}^{L}$, is related to that in the global system, p, through

$$
\mathbf{p}^{L}=\mathbf{T p}
$$

The particular formulation of the phase-field model given in Eq. (7) encodes traction free, electrically permeable, and free polarization boundary conditions on the crack faces. For a sharp crack, the traction-free conditions imply that the mechanical traction vanishes on the crack faces. The permeable crack conditions assume that the crack does not perturb the electrical fields, i.e. the crack does not exist electrically. These conditions have been
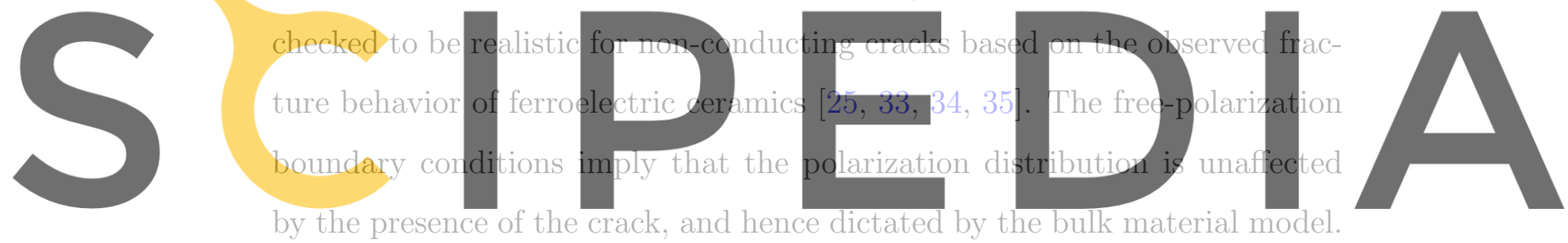

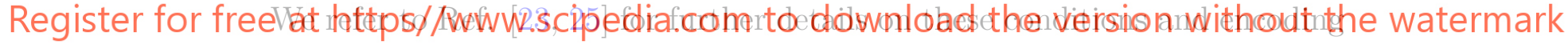

them in the phase-field framework.

The evolution of the ferroelectric domain microstructure and the fracture results from the gradient flows of the total electro-mechanical enthalpy in Eq. (4), with Eqs. (5) and (7), based on the time-dependent GinzburgLandau (TDGL) equations. Here, the primary order variables are $\mathbf{p}$ and $v$ for the microstructure and fracture processes, respectively. Thus, the governing equations are obtained by assuming that the displacement and the electric field adjust immediately to mechanical and electrostatic equilibrium (with 
infinite mobility), i.e.

$$
\begin{aligned}
\mu_{p} \int_{\Omega} \dot{p}_{i} \delta p_{i} \mathrm{~d} \Omega= & -\int_{\Omega} \frac{\partial h^{\text {poly }}}{\partial p_{i}} \delta p_{i} \mathrm{~d} \Omega \\
\mu_{v} \int_{\Omega} \dot{v} \delta v \mathrm{~d} \Omega= & -\int_{\Omega} \frac{\partial h^{\text {poly }}}{\partial v} \delta v \mathrm{~d} \Omega \\
& -2 G_{c} \int_{\Omega}\left(\frac{v-1}{4 \kappa} \delta v+\kappa v_{, i} \delta v_{, i}\right) \mathrm{d} \Omega \\
0 & \int_{\Omega} \sigma_{i j} \delta \varepsilon_{i j} \mathrm{~d} \Omega \\
0= & \int_{\Omega} D_{i} \delta E_{i} \mathrm{~d} \Omega
\end{aligned}
$$

where $1 / \mu_{p}>0$ and $1 / \mu_{v}>0$ are the mobilities of the processes. The stresses and electric displacements are derived from the enthalpy density, respectively, as $\sigma=\partial h^{\text {poly }} / \partial \varepsilon$ and $\mathbf{D}=-\partial h^{\text {poly }} / \partial \mathbf{E}$. The mechanical strain $\varepsilon$ and the electric field $\mathbf{E}$ are associated with the mechanical displacement $\mathbf{u}$ and the electric potential $\phi$, respectively as $\varepsilon=1 / 2\left(\nabla \mathbf{u}+\nabla^{T} \mathbf{u}\right)$ and $\mathbf{E}=-\nabla \phi$.

The weak forms of the evolution and equilibrium equations are discretized in space with standard finite elements. Equations (13) and (14) are discretized in time with a semi-implicit scheme. Note that the minimization in Eq. (15) is non-smooth, and a quasi-Newton algorithm is applied to solve the mechanical equilibrium problem following [32].

A simple algorithm to solve the coupled system in a straightforward staggered approach is presented next. This algorithm describes how to advance in one load step $w^{n}$, and it is meant to achieve steady states for both ferroelectric domains and fracture processes in each load step. The functions $\mathbf{g}\left(w^{n}\right)$ and $f\left(w^{n}\right)$ encode the Dirichlet data for the mechanical displacement and electric potential as a function of the load step. After reaching a steady state for both the polarization $\mathbf{p}$ and the phase-field $v$ with small tolerances 
$\delta_{\text {ferro }}$ and $\delta_{v \text { field }}$, respectively, the values for $v^{n}, \mathbf{u}^{n}, \mathbf{p}^{n}$ and $\phi^{n}$ are recorded and the algorithm proceeds to a new load step $w^{n+1}$. Since the crack should not be allowed to heal, it is necessary to supplement the model with an irreversibility condition, implying that the field $v$ should be a monotonically decreasing function of time. This is an inequality condition which is numerically cumbersome to implement. In practice, this condition can be replaced by freezing the field $v$ to 0 wherever it reaches a given small threshold $\zeta$ in each load step [36, 37].

We note that it is possible to induce a negative crack driving force in electromechanical fracture. The model then favors values of $v$ much larger than one in localized regions, which is not physically meaningful. This issue manifests itself with negative energy release rates in sharp crack models of linear piezoelectric materials [7]. We deal with this issue by limiting the maximum value of $v$ to 1 .

\section{Numerical simulations and discussion}

For numerical simulations, a cubic domain is considered with the boundary conditions depicted in Fig. A.1. The normalized dimensions of the domain are $200 \times 200 \times 200$ according to the normalizations presented in Appendix A. It is discretized with approximately $6,000,000$ tetrahedral finite elements of size $h \simeq 2$. First, the phase-field model of grain growth described in Section 2.1 is used to obtain different polycrystalline microstructures. The parameters of the kinetic equation (3) are selected as $\alpha=1$, $\beta=1, \gamma=1, \kappa_{i}=5$ and $\mu_{g}=1$. The time step $\Delta t=2 \times 10^{-2}$ is also chosen for the numerical integration scheme. Forty field variables 


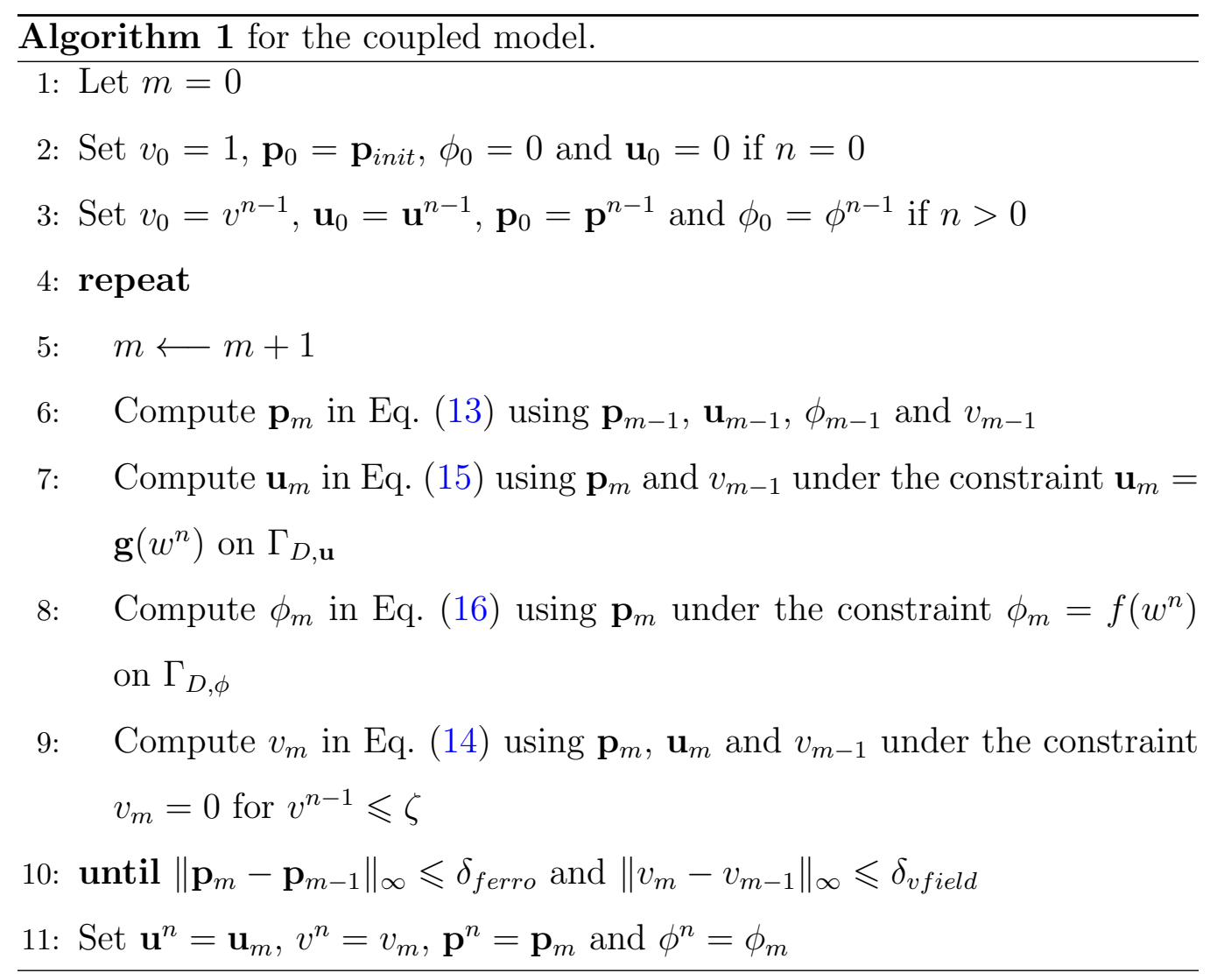


$(m=40)$ are considered and they are initialized with random small variables as $-0.001<\eta_{i}(i=1, \ldots, 40)<0.001$. The function $\mathscr{F}$ in Eq. (5) is chosen to be proportional to $\xi$ as $\mathscr{F}(\xi)=\xi$. The power of $\xi$ is set to $k=1$ to prevent the ratio of the fracture toughness along the grain boundaries over that of the grain interior from reaching unphysically low values [38].

The simulations were performed in the large-scale computing facility Marenostrum at the Barcelona Supercomputing Center using up to 512 processors for 19000 steps with the finite element library of the Kratos multiphysics package [39]. The contour of $\xi$ in Eq. (6) is presented in Fig. A.2 for two snapshots of the polycrystalline microstructure evolution. The grain boundaries correspond to darker regions in this figure. In the early stages of the grain growth simulation, the field variables $\eta_{i}$ start to grow at different locations of the sample. After about 2500 steps, a well defined microstructure consisting of about 55 grains can be observed in Fig. A.2(a). Further evolution of the model leads to grain growth and coalescence. A coarse-grain microstructure is obtained at the end of the simulation, which is depicted in Fig. A.2(b).

The obtained microstructures presented in Figs. A.2(a) and A.2(b) are used for the fracture simulations of the fine- and coarse-grain ferroelectric polycrystals, respectively. The function $\xi$ attains its lowest value - about 0.2 - at the grain boundaries for both microstructures. This value is the ratio of the critical fracture energy of the grain boundary to that of the grain interior in our model. The grain orientations $\Phi_{i}, \Theta_{i}$, and $\Psi_{i}(i=1, \ldots, 40)$, are assigned randomly between $0^{\circ}$ and $45^{\circ}$ using the functions $\phi, \theta$, and $\psi$ in Eq. (10). Figure A.3 presents a sample of the distribution of the grain 
orientation $\theta$ over the fine-grain microstructure.

For the fracture simulations, a monotonically increasing mechanical load is applied by pulling the top and bottom faces of the model with a uniform mechanical displacement such that $\mathbf{u}_{ \pm}=(0, \pm w, 0)$, where + and - indicate the top and bottom faces of the model respectively and $w$ is the load step. To propitiate the crack initiation, the $x_{2}$-component of the mechanical displacement is also fixed to a certain value at the top half and bottom half of the front face highlighted with yellow in Fig. A.1, so that $u_{2 \pm}= \pm w$. Since the computational domain represents a piece of a larger ferroelectric polycrystal, we should avoid lateral compression of the model in the $x_{3}$ direction due to the applied mechanical loading. For this purpose, the $x_{3}$-component of the mechanical displacement is fixed on the right (highlighted with green) and left faces. The normalized initial polarization $\mathbf{p}_{\text {init }}=(1,0,0)$ is assigned along the positive $x_{1}$-direction in the global coordinate system, see Fig. A.1. Since grain orientations are chosen between $0^{\circ}$ and $45^{\circ}$, the local initial polarization in each grain is mainly along the local $x_{1}$-direction. In this way, the ferroelastic switching becomes favorable with an acceptable mismatch between the grains, consequently leading to strong interactions between the microstructure and the crack propagation. As for the electrical boundary conditions, the electric potential on the front (yellow) and back faces is set to 0 . It is also assumed that the normal component of the electric displacement vanishes on the other faces and the free-polarization boundary conditions are satisfied at these faces. The simulation constants are chosen as follows. The tolerances to achieve steady states for ferroelectric domains and fracture

processes are set to $\delta_{\text {ferro }}=5 \times 10^{-3}, \delta_{\text {vfield }}=2 \times 10^{-3}$, the threshold to 
detect the irreversibly fractured regions to $\zeta=2 \times 10^{-2}$, the inverse mobilities to $\mu_{p}=1$ and $\mu_{v}=15$, the residual stiffness to $\eta_{\kappa}=10^{-4}$ and the regularization parameter to twice the element size as $\kappa=4$. Three hundred load steps are considered in the simulation $(n=300)$ with load increments of $\Delta w^{n}=10^{-2}$. A normalized time step of $\Delta t_{m}^{\prime}=0.1$ leads to convergent and accurate solutions for the semi-implicit integration of the gradient flow equations.

As the load increases, the field $v$ starts to decrease around the center-left line of the sample (dashed line in Fig. A.1) until it reaches the threshold to be considered permanently fractured $(v<0.02)$ at load step $n=50$ and the crack initiates. By further increasing the load during the following load steps, the crack propagates into the sample. To visualize the 3-D fracture evolution, we plot the isosurfaces of the fracture parameter $v$ for a positive value between 0 and 1 . This value is chosen as $v=0.5$ to clearly show a zone encompassing the fracture. Figure A.4 presents four snapshots of the fracture evolution in the fine-grain microstructure. The crack cuts the sample into two parts at load step $\mathbf{d}$, creating a deflected and complex fracture surface. The crack deflection is due to the interaction between the crack and the polycrystalline microstructure. Since the fracture toughness of the grain interior is higher than that of the grain boundary, the crack propagation is more favorable along the grain boundary - inter-granular crack propagation mode- than in the grain interior - trans-granular crack propagation modeleading to the "zig-zag" fracture pattern. To distinguish the inter- and transgranular crack propagation, the isosurfaces of parameters $z=(1-v)(1-\xi)$ and $z^{\prime}=(1-v) \xi$ are presented in Fig. A.5. These figures are obtained after 
the samples are splitted into two parts for both the fine- and the coarse-grain structures. The parameter $z$ is positive when the crack propagates through the grain boundary, i.e. $v$ and $\xi$ are close to zero, while the parameter $z^{\prime}$ is positive when the crack propagates through the grain interior, i.e. $v$ is close to zero and $\xi$ is close to one. Therefore the parameters $z$ and $z^{\prime}$ represent the inter- and trans-granular modes of fracture, respectively. It is obvious in Fig. A.5 that the trans-granular crack patterns in both structures are nearly flat in comparison with the rugged inter-granular patterns.

To evaluate the effect of the fracture toughening mechanisms, the evolution of the normalized surface energy (the second integral in Eq. (4)) is presented in Fig. A.6 as a function of the load step $n$. Note that the surface energy is an indirect measure of the crack area. The letters of the fine-grain graph indicate instants of the simulation corresponding to the snapshots shown in Fig. A.4. Both the fine- and coarse-grain energy graphs start from zero (pristine sample), and are almost identical until the cracks initiate at load step $n=50$. After this point, the crack in the fine-grain structure starts to propagate faster along the grain boundaries, while the crack in the coarse-grain structure starts to cut the grains, and hence propagates slower. Considering the low fracture toughness of the grain boundaries, the intergranular propagation of cracks in the fine-grain structure should be abrupt, i.e. a small increase of the load should lead to a big jump in the surface energy or the crack area. However, reported toughening mechanisms hinder this behavior. One of these mechanisms, crack branching, is shown in Fig. A.7. After the initiation, the crack propagates slowly along the upper branch, indicated by the white arrow, until load step b. After this point, a secondary 
crack initiates along the lower grain boundary and propagates until the total failure of the sample at load step $\mathbf{d}$. Crack branching thus partly explains the slower crack propagation observed until load step b. Other associated toughening mechanisms are explained next.

Figure A.6 shows another slow crack propagation interval between load steps $\mathbf{b}$ and $\mathbf{c}$ in the fine-grain structure. Figure A.8 presents the crack configuration at load step c. The nucleation of an isolated crack, indicated with the white arrow, is apparent in this figure. The grain highlighted with yellow acts as a bridge between the main and isolated cracks, preventing their coalescence. Therefore, crack bridging acts as a fracture toughening mechanism in polycrystals. Interestingly, this mechanism has been also observed in experiments $[28,40,41]$.

Crack deflection is an additional toughening mechanism in ferroelectric polycrystals. As presented in Fig. A.5, the inter-granular crack creates a deflected fracture pattern. Crack deflection occurs when the crack meets a tough grain ahead and it is forced to move around the grain since the fracture toughness of the grain interior is higher. This phenomenon leads to an increase in the crack area, and hence an increase in the dissipated energy. Crack deflection toughening slows down the crack growth in both fine- and coarse-grain structures.

In addition to the interaction with the polycrystalline microstructure, the cracks also interact with the ferroelectric domain microstructure. Figure A.9 presents three snapshots of the evolution of the ferroelectric microstructure at different load steps in the coarse-grain structure. The tetragonal variants are distinguished based on the polarization in the local grain coordinate system 
and presented by colors following an illustratation method of domain states in phase-field models of ferroelectric polycrystals [42, 43].

Due to the high tensile stresses around the fracture zone, the polarization vectors start to rotate mainly towards the vertical $x_{2}$-direction, leading to $90^{\circ}$ ferroelastic domain switching. The formation of a multiple-domain structure is apparent in Fig. A.9(b). The switched regions form twins around the crack tip. Since the crystal's unit cell is longer along the polarization direction, as encoded in the Landau-Devonshire model, compressive stresses are induced by the twins, leading to a strong toughening effect, already pointed out in the literature [2]. $90^{\circ}$ ferroelastic domain switching is also favorable in the grains with a high misalignment since the effect of the misalignment has some similarity with the application of stress [44]. In fact, the mismatch at the grain boundary gives rise to internal stresses, facilitating the alignment of the polarization upon switching with the vertical $x_{2}$-direction under tensile loading. This leads to the formation of multiple twins around the crack shielding the crack tip from the applied loading. As the load increases, these twins grow and a new set of twins nucleates in unswitched regions, compare Figs. A.9(b) and A.9(c). For clarification purposes, the right view of Fig. A.9(c) is presented in Fig. A.10, where the polarization vectors in the global coordinate system are also depicted. The slow crack propagation period, observed from the load step a in the energy graph of Fig. A.6 (Coarse-grain), is due to a considerable expansion of $90^{\circ}$ switched domains, presented in Fig. A.9(c). This toughening mechanism is also reported in experiments of crack propagation in $\mathrm{BaTiO}_{3}[28,40,41,45]$. Note that the ferroelastic switching is less pronounced in the fine-grain structure, since the crack area 
increases faster for low applied load as compared to the coarse-grain case, as shown in the crack area evolution in Fig. A.6.

\section{Conclusions}

We perform, to the best of our knowledge, the first three-dimensional simulations of crack growth in ferroelectric polycrystals with the goal of qualitatively evaluating the effect of the grain and ferroelectric domain microstructures on the fracture process. The simulations are based on coupling three phase-field models describing (1) the poly-crystalline structure, (2) the location of the cracks and (3) the ferroelectric domain microstructure. The model captures the physical complexity of the fracture processes in this type of materials, and in particular the complex interactions between the three interfaces, the latter two of them evolving. The simulation results show that crack deflection, crack bridging, crack branching and ferroelastic domain switching act as the main fracture toughening mechanisms. These mechanisms are intrinsically three-dimensional and our fully 3-D simulations illustrate how the combination of all of them enhances the macroscopic fracture toughness of the material. All of these toughening mechanisms have been observed in experiments of ferroelectric ceramics [28, 40, 41, 45]. Thus, this study constitutes a proof of concept of the model and the simulation methodology, adequate for qualitative assessment. The present work also paves the path for further studies probing systematically the fracture behavior of ferroeletric polycrystals. For instance, a micromechanics approach based on representative volume elements can be envisioned to extract the effective properties for macroscopic phenomenological models. 
However, more work is needed for quantitative evaluation of the combined effect of the polycrystalline microstructure and the ferroelectric domain microstructure on the fracture response of ferroelectric polycrystals. In particular, the relative physical size of the grains and ferroelectric domains should be considered for more realistic - but extremely demanding from a computational viewpoint - simulations. A detailed experimental characterization of the grain boundaries would be desirable, including their width as well as the dependence of the grain boundaries' properties on the crystallographic misorientation of the neighboring grains. The width of the domain walls can also be determined experimentally [46]. The width of the smeared cracks, however, is a numerical artifact, which could have a significant effect on the simulation results. A sensitivity analysis on the regularization parameter of the fracture phase-field relative to the other lengthscales of the problem, i.e. width of the grain boundaries and width of the domain wall, would be necessary. Another important issue is the quantification and relative magnitude of the parameters $\mu_{p}$ and $\mu_{v}$, which can have an important effect on the resulting response.

More fundamental research is also needed to characterize the fracture behavior of ferroelectric ceramics, and properly model it in the phase-field context. Here, we have assumed that the fracture toughness does not depend on orientation, and considered a fracture toughness measured for an annealed sample. The quantitative mapping between the experimental measurements in these or other conditions and the model parameters requires further work. Also, the intrinsic anisotropy of the crystal, i.e. the different surface energy in different cleavage planes, is bound to have an effect. Furthermore, in our 
model the tension-compression behavior is determined relative to a reference cubic phase, but it seems more reasonable to refer it to the closest free energy well (variant). Thus, many open questions remain, which demand tackling this problem with a variety of experimental and theoretical methods.

\section{Acknowledgements}

The authors gratefully acknowledge insightful comments of an anonymous referee, and the support of the Ministerio de Ciencia e Innovación (DPI2011-26589), and the computer resources, technical expertise and assistance provided by the Barcelona Supercomputing Center - Centro Nacional de Supercomputación.

\section{References}

[1] Zhang TY, Gao CF. Theor Appl Fract Mech 2004;41:339.

[2] Schneider GA. Annu Rev Mater Res 2007;37:491.

[3] Kuna M. Eng Fract Mech 2010;77:309.

[4] McMeeking RM. Eng Fract Mech 1999;64:217.

[5] McMeeking RM. Eng Fract Mech 2004;71:1149.

[6] Landis CM. Int J Solids Struct 2004;41:6291.

[7] Li WY, McMeeking RM, Landis CM. Eur J Mech A-Solids 2008;27:285.

[8] Wang J, Landis CM. J Mech Mater Struct 2006;1:1075.

[9] Sheng JS, Landis CM. Int J Fract 2007;143:161. 
[10] Hwang SC, Lynch CS, McMeeking RM. Acta Mater 1995;43:2073.

[11] Zhu T, Yang W. Acta Mater 1997;45:4695.

[12] Yang W, Zhu T. J Mech Phys Solids 1998;46:291.

[13] Zhu T, Yang W. J Mech Phys Solids 1999;47:81.

[14] Beom HG, Atlurib SN. J Mech Phys Solids 2003;51:1107.

[15] Song YC, Soh AK, Ni Y. J Phys D: Appl Phys 2007;40:1175.

[16] Wang J, Zhang TY. Acta Mater 2007;55:2465.

[17] Wang J, Kamlah M. Smart Mater Struct 2009;18:104008.

[18] Xu BX, Schrade D, Gross D, Mueller R. Int J Fract 2010;165:163.

[19] Li W, Landis CM. Eng Fract Mech 2011;78:1505.

[20] Landis CM. J Mech Phys Solids 2003;51:1347.

[21] Li Q, Kuna M. Comput Mater Sci 2012;57:94.

[22] Verhoosel CV, Gutierrez MA. Eng Fract Mech 2009;76:742.

[23] Abdollahi A, Arias I. Acta Mater 2011;59:4733.

[24] Abdollahi A, Arias I. Model Simul Mater Sci Eng 2011;19:074010.

[25] Abdollahi A, Arias I. J Mech Phys Solids 2012;60:2100.

[26] Abdollahi A, Arias I. Acta Mater 2013;DOI: http://dx.doi.org/10.1016/j.actamat.2013.07.050. 
[27] Abdollahi A, Arias I. Int J Fract 2012;174:3.

[28] Meschke F, Kolleck A, Schneider GA. J Eur Ceram Soc 1997;17:1143.

[29] Fan D, Chen LQ. Acta Mater 1997;45:611.

[30] Krill CE, Chen LQ. Acta Mater 2002;50:3057.

[31] Griffith AA. Philos Trans Royal Soc London 1921;A221:163.

[32] Amor H, Marigo JJ, Maurini C. J Mech Phys Solids 2009;57:1209.

[33] Schneider GA, Felten F, McMeeking RM. Acta Mater 2003;51:2235.

[34] Haeusler C, Jelitto H, Neumeister P, Balke H, Schneider GA. Int J Fract 2009;160:43.

[35] Engert AR, Neumeister P, Mecklenburg M, Jelitto H, Balke H, Schneider GA. J Eur Ceram Soc 2011;31:531.

[36] Bourdin B. Interfaces Free Bound 2007;9:411.

[37] Bourdin B, Francfort GA, Marigo JJ. J Elast 2008;91:5.

[38] Sukumar N, Srolovitz DJ, Baker TJ, Prevost JH. Int J Numer Meth Eng 2003;56:2015.

[39] Dadvand P, Rossi R, Onate E. Arch Comput Methods Eng 2010;17:253.

[40] Meschke F, Raddatz O, Kolleck A, Schneider GA. J Am Ceram Soc 2000;83:353. 
[41] Forderreuther A, Thurn G, Zimmermann A, Aldinger F. J Eur Ceram Soc 2002;22(12):2023.

[42] Choudhury S, Li YL, 111 CEK, Chen LQ. Acta Mater 2005;53:5313-21.

[43] Choudhury S, Li YL, 111 CEK, Chen LQ. Acta Mater 2007;55:1415-26.

[44] Zhang W, Bhattacharya K. Acta Mater 2005;53(1):199-209.

[45] Fang DN, Jiang YJ, Li S, Sun CT. Acta Mater 2007;55:5758.

[46] Stemmer S, Streiffer SK, Ernst F, Ruhle M. Phil Mag A 1995;71:713.

[47] Devonshire AF. Philos Mag 1949;40:1040.

[48] Devonshire AF. Philos Mag 1951;42:1065.

[49] Su Y, Landis CM. J Mech Phys Solids 2007;55:280.

[50] Shu YC, Bhattacharya K. Philos Mag B 2001;81(12):2021.

[51] Zhang W, Bhattacharya K. Acta Mater 2005;53:185.

[52] Floquet N, Valot CM, Mesnier MT, Niepce JC, Normand L, Thorel A, et al. J Phys III 1997;7:1105. 


\section{Appendix A. Energy functions and material constants for $\mathrm{BaTiO}_{3}$}

The energy functions $U, W$, and $\chi^{\text {poly }}$ in Eq. (9) are chosen following $[47,48]$, expanded in terms of three-dimensional components as [49]

$$
\begin{aligned}
U\left(p_{i, j}\right)=\frac{a_{0}}{2}\left(p_{1,1}^{2}+p_{2,2}^{2}+p_{3,3}^{2}+p_{1,2}^{2}+p_{2,1}^{2}+p_{1,3}^{2}+p_{3,1}^{2}+p_{2,3}^{2}+p_{3,2}^{2}\right), \\
\begin{aligned}
W\left(p_{i}, \varepsilon_{j k}\right)= & -\frac{b_{1}}{2}\left(\varepsilon_{11} p_{1}^{2}+\varepsilon_{22} p_{2}^{2}+\varepsilon_{33} p_{3}^{2}\right) \\
& -\frac{b_{2}}{2}\left(\left(\varepsilon_{22}+\varepsilon_{33}\right) p_{1}^{2}+\left(\varepsilon_{11}+\varepsilon_{33}\right) p_{2}^{2}+\left(\varepsilon_{11}+\varepsilon_{22}\right) p_{3}^{2}\right) \\
& -b_{3}\left(\left(\varepsilon_{12}+\varepsilon_{21}\right) p_{1} p_{2}+\left(\varepsilon_{13}+\varepsilon_{31}\right) p_{1} p_{3}+\left(\varepsilon_{23}+\varepsilon_{32}\right) p_{2} p_{3}\right)
\end{aligned}
\end{aligned}
$$

$$
\begin{aligned}
\chi^{\text {poly }}\left(p_{i}\right)=\chi\left(p_{i}^{L}\right)= & \frac{a_{1}}{2}\left[\left(p_{1}^{L}\right)^{2}+\left(p_{2}^{L}\right)^{2}+\left(p_{3}^{L}\right)^{2}\right]+\frac{a_{2}}{4}\left[\left(p_{1}^{L}\right)^{4}+\left(p_{2}^{L}\right)^{4}+\left(p_{3}^{L}\right)^{4}\right] \\
& +\frac{a_{3}}{2}\left[\left(p_{1}^{L} p_{2}^{L}\right)^{2}+\left(p_{2}^{L} p_{3}^{L}\right)^{2}+\left(p_{1}^{L} p_{3}^{L}\right)^{2}\right] \\
& +\frac{a_{4}}{6}\left[\left(p_{1}^{L}\right)^{6}+\left(p_{2}^{L}\right)^{6}+\left(p_{3}^{L}\right)^{6}\right] \\
& +\frac{a_{5}}{4}\left[\left(p_{1}^{L} p_{2}^{L}\right)^{4}+\left(p_{2}^{L} p_{3}^{L}\right)^{4}+\left(p_{1}^{L} p_{3}^{L}\right)^{4}\right]
\end{aligned}
$$

where $a_{0}$ is the scaling parameter of the domain wall energy, $a_{i}(i=1, . ., 5)$ are the constants of the phase separation energy and $b_{i}(i=1,2,3)$ are the constants of the electro-mechanical coupling terms. The local polarization $\mathbf{p}^{L}$ is related to the global polarization $\mathbf{p}$ through Eq. (12). For convenience, dimensionless variables are selected through the following normalizations: $x_{i}^{\prime}=x_{i} \sqrt{c_{0} / a_{0}} / p_{0}, \mu^{\prime}=\mu / c_{0}, \kappa_{0}^{\prime}=\kappa_{0} / c_{0}, p_{i}^{\prime}=p_{i} / p_{0}, t^{\prime}=t c_{0} / \mu p_{0}^{2}, \varepsilon_{0}^{\prime}=$ $\varepsilon_{0} c_{0} / p_{0}^{2}, \phi^{\prime}=\phi / \sqrt{a_{0} c_{0}}, a_{1}^{\prime}=a_{1} p_{0}^{2} / c_{0}, a_{2}^{\prime}=a_{2} p_{0}^{4} / c_{0}, a_{3}^{\prime}=a_{3} p_{0}^{4} / c_{0}, a_{4}^{\prime}=$ 
$a_{4} p_{0}^{6} / c_{0}, a_{5}^{\prime}=a_{5} p_{0}^{8} / c_{0}$, and $b_{i}^{\prime}=b_{i} p_{0}^{2} / c_{0}$, where $i=1,2,3$. The equations with normalized variables are the same as the original ones. The constants are chosen to fit the behavior of single crystals of barium titanate $\left(\mathrm{BaTiO}_{3}\right)$ at room temperature, taking $c_{0}=1 \mathrm{GPa}$ and a value for the spontaneous polarization of $p_{0}=0.26 \mathrm{Cm}^{-2}$. The electro-mechanical coupling constants $b_{i}$ $(i=1,2,3)$ are obtained considering the spontaneous strains of the tetragonal phase $\varepsilon_{a}=-0.44 \%$ along a-axis and $\varepsilon_{c}=0.65 \%$ along c-axis, with respect to the paraelectric cubic phase, which is the reference configuration of the model $[50,51]$. The domain wall scaling parameter is set to $a_{0}=3.7 \times 10^{-9} \mathrm{Vm}^{3} \mathrm{C}^{-1}$ which leads to the value of 0.5 nanometer for the normalized unit length $\Delta x^{\prime}$ $=1$. By setting the normalized scaling parameter of the domain wall energy to $a_{0}^{\prime}=0.2$, the normalized width of domain walls is obtained between 6 and 8 in the simulations, corresponding to 3-4 nanometers, in the order of experimentally measured values in tetragonal ferroelectric ceramics [46, 52]. Note that to simplify the numerical procedure applied for the model solution, the electrostrictive coefficients $b_{i}(i=1,2,3)$ are assumed to be isotropic. This assumption is unlikely to affect the general behavior of the model. The intrinsic fracture toughness of $\mathrm{BaTiO}_{3}$ is taken as $K_{c}=0.49 \mathrm{MPa} \sqrt{m}$ from experimental measurements on an annealed sample [40]. Considering Young's modulus and Poisson's ratio for $\mathrm{BaTiO}_{3}$ as $E=100 \mathrm{GPa}$ and $\nu=0.37$ respectively (consistent with the bulk and shear modulus), the value of the critical energy release rate is obtained as $G_{c}=\left(1-\nu^{2}\right) K_{c}^{2} / E=2 \mathrm{Jm}^{-2}$. The value of normalized critical energy release rate is then calculated as $G_{c}^{\prime}=G_{c} \sqrt{1 / a_{0} c_{0}} / p_{0}=4$. The normalized parameters are presented in Table A.1. 


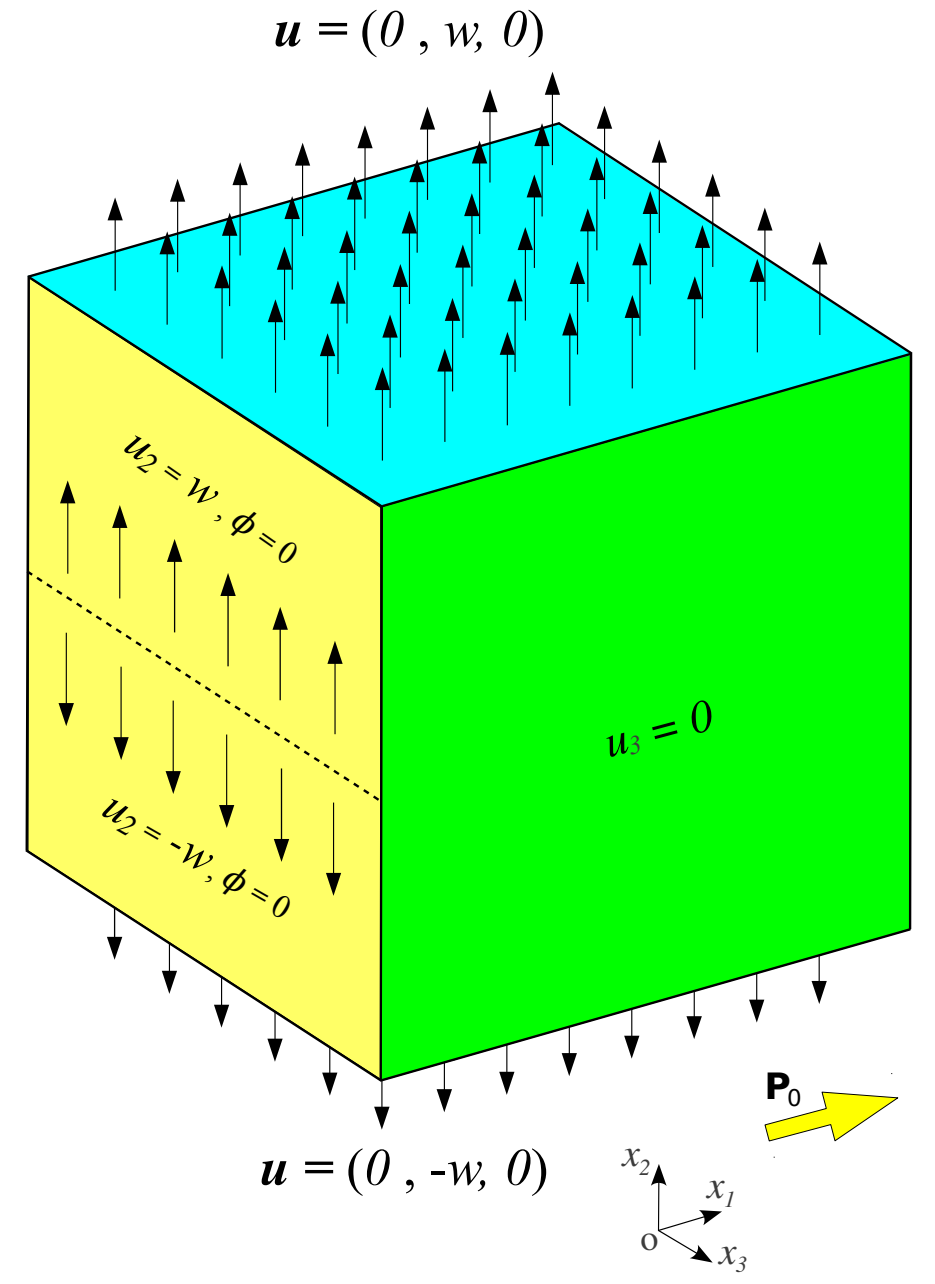

Figure A.1: 3-D computational model with boundary conditions. 




Figure A.2: Two snapshots of the 3-D polycrystalline microstructure evolution at time steps (a) $t=2500$ (b) $t=19000$. The gray contour indicates the distribution of $\xi$. Grain boundaries correspond to darker regions. These two microstructures are considered later, representative of a fine and a coarse-grained polycrystal, respectively. 


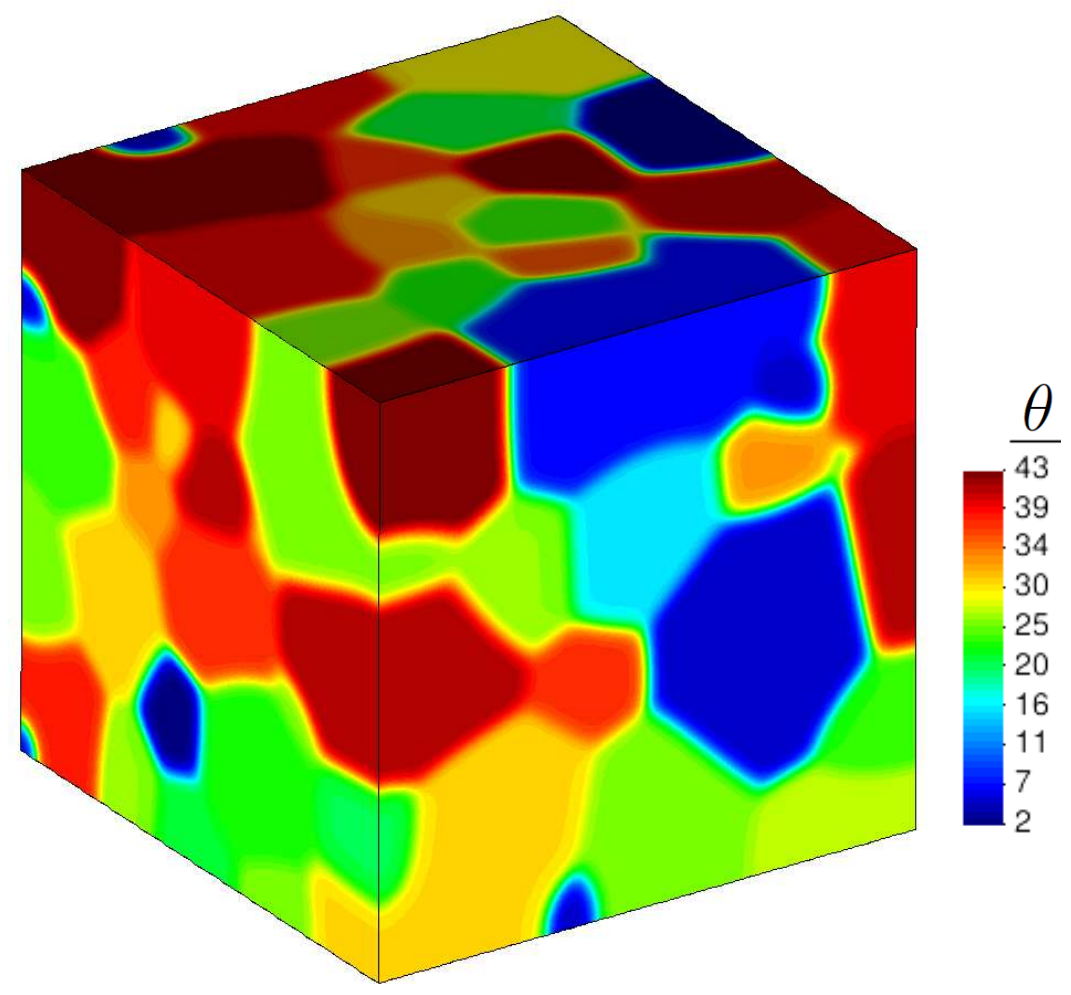

Figure A.3: Distribution of the rotation field $\theta$ in Eq. 10 over the fine-grain microstructure presented in Fig. A.2(a). 

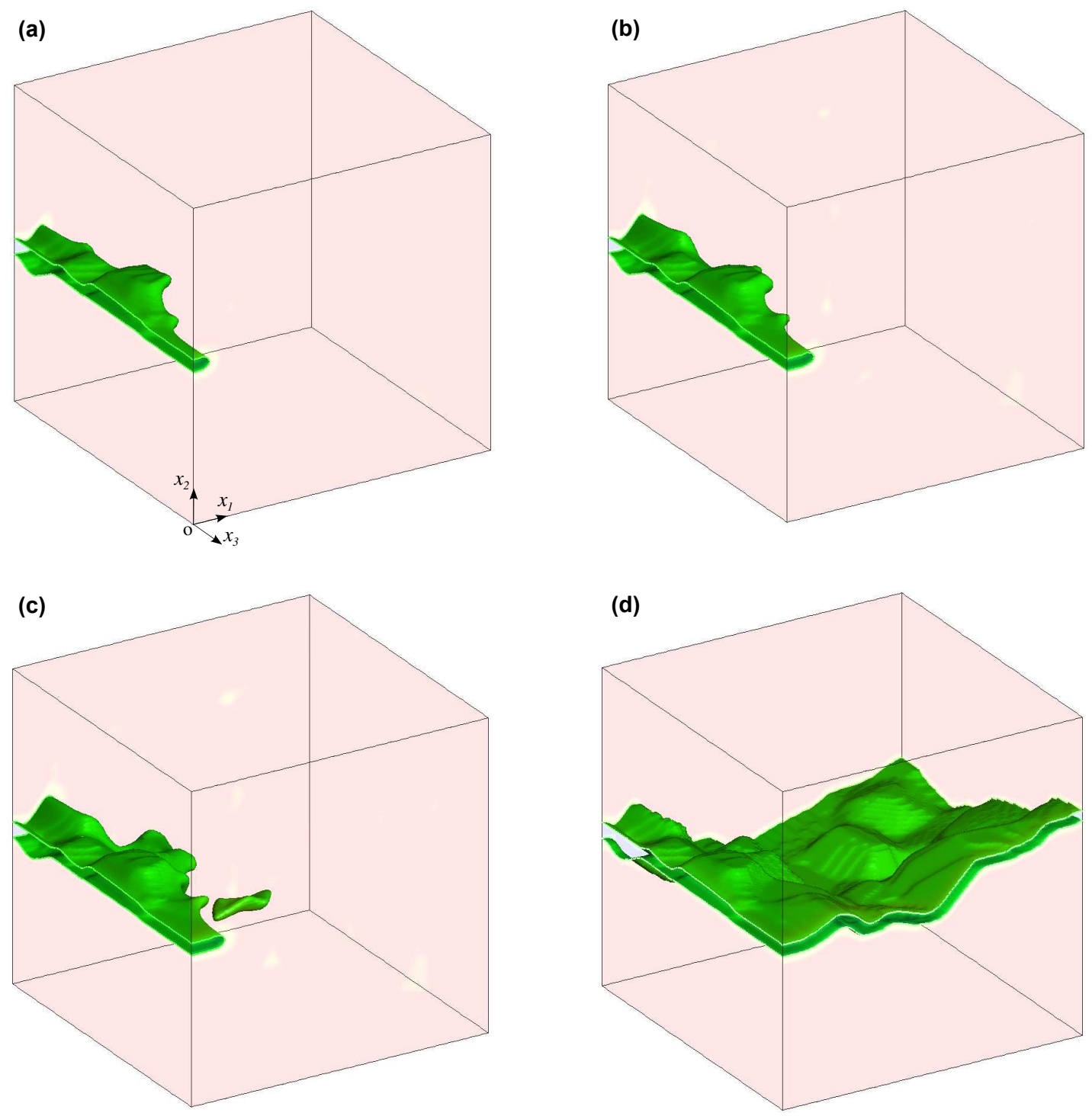

Figure A.4: Four snapshots of the fracture evolution in the fine-grain microstructure at load steps (a) $n=120$ (b) $n=150$ (c) $n=160$ (d) $n=170$. The green surface is the isosurface of the fracture parameter for the value of $v=0.5$. 

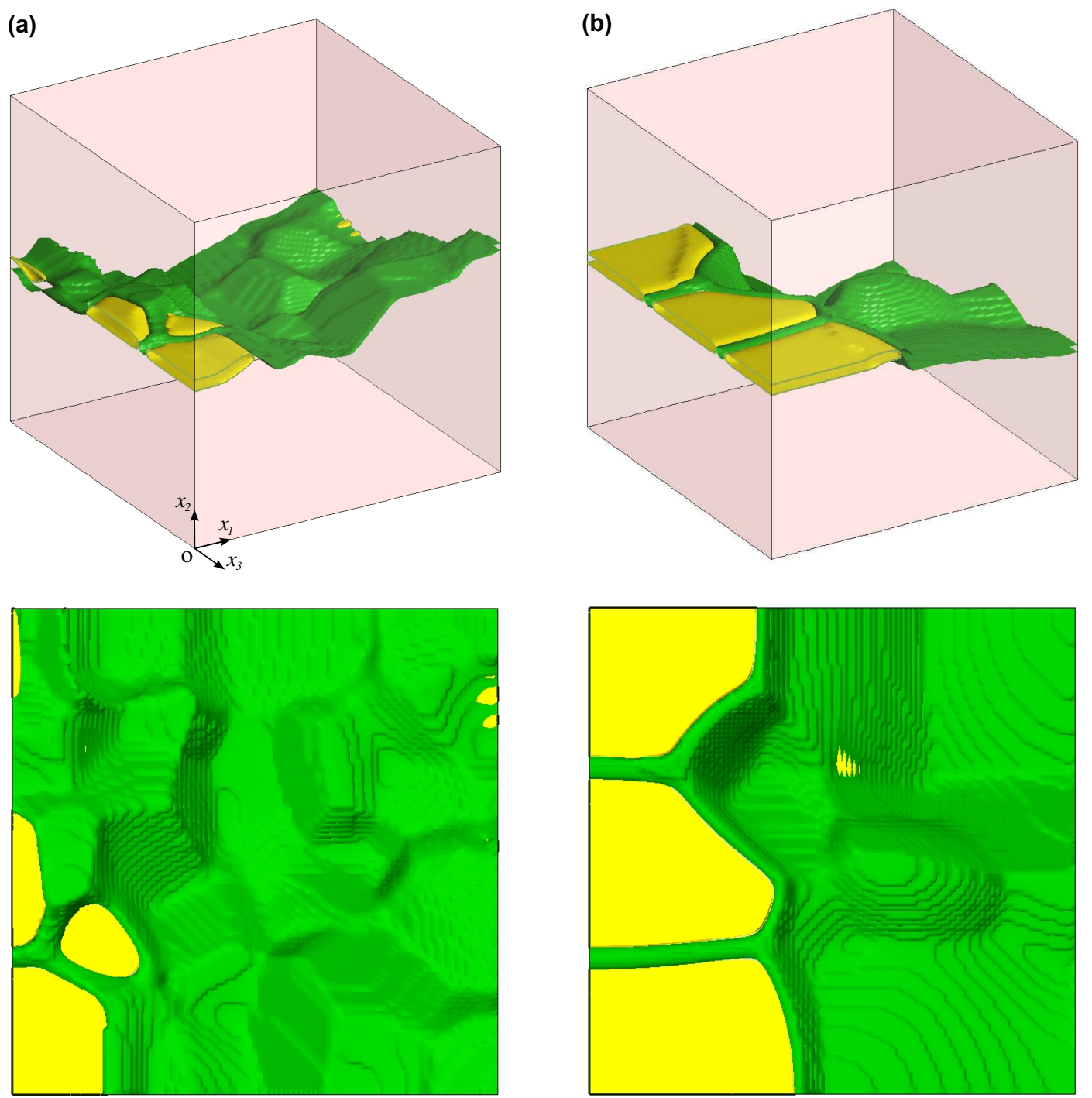

Figure A.5: Isosurface of the parameters $z=(1-v)(1-\xi)$ and $z^{\prime}=(1-v) \xi$, indicating the inter- and trans-granular crack surfaces highlighted with green and yellow, respectively for (a) fine-grain and (b) coarse-grain microstructures. The bottom figure in each case presents the top view of the isosurface. 


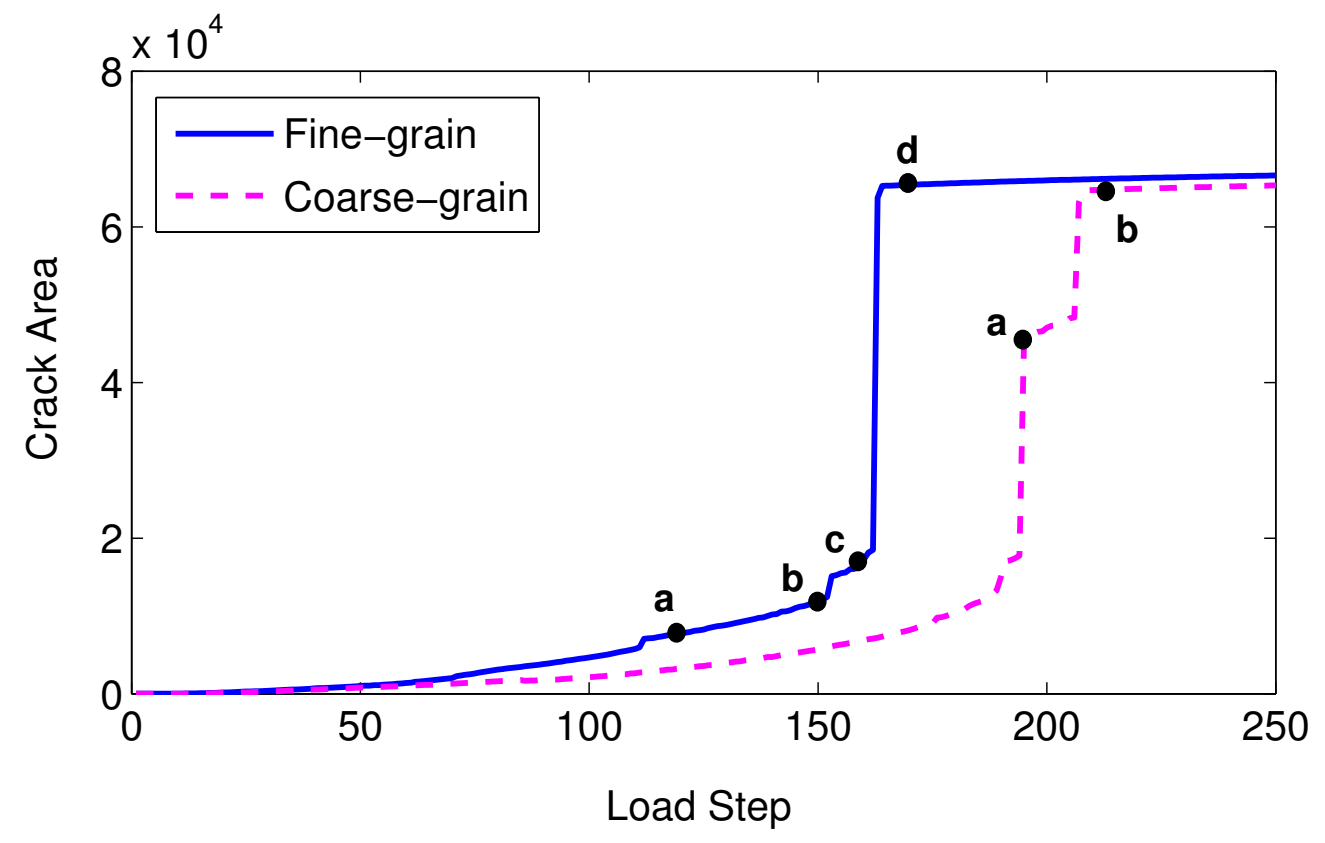

Figure A.6: Evolution of the normalized crack area as a function of the load step in fineand coarse-grain microstructures. 


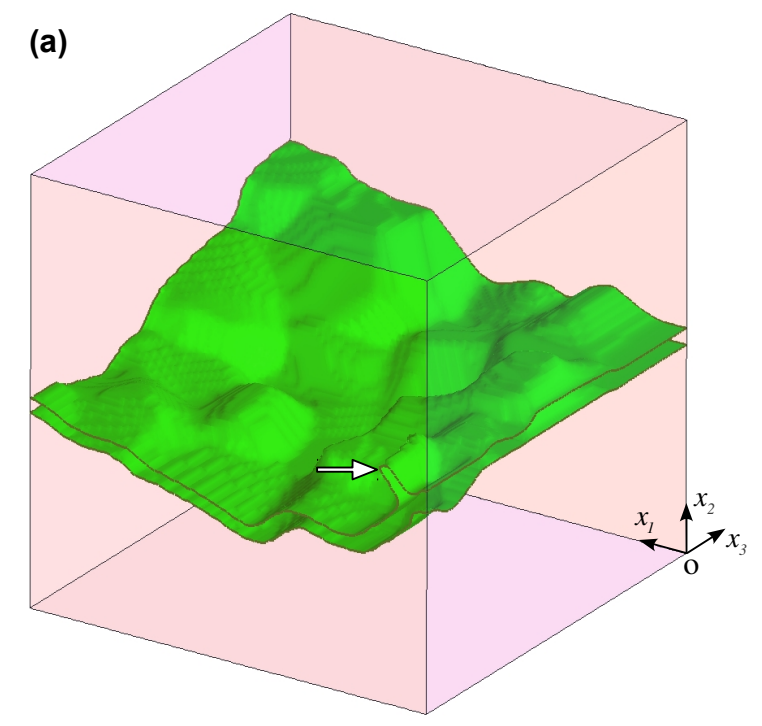

(b)

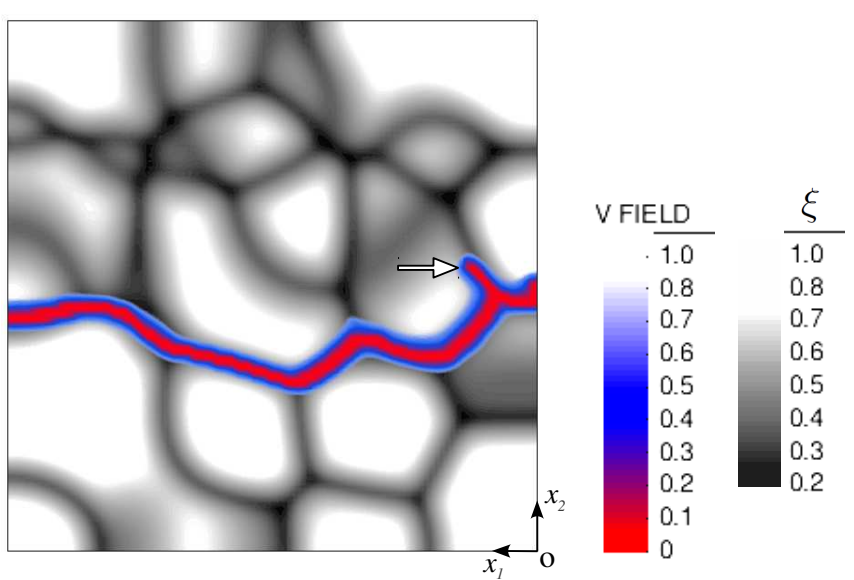

Figure A.7: Crack branching in the fine-grain microstructure: (a) isosurface of the fracture parameter $v$ (b) distribution of $v$ (colour contour) and $\xi$ (gray contour), where grain boundaries and fracture zone are highlighted with the darker and red regions, respectively, and the area where $v>0.8$ is transparent to allow the visualization of the polycrystalline microstructure. The white arrow indicates the first crack branch. Figure (b) presents the contours in a cross section of the 3-D model which cuts the two crack branches with the surface unit normal $(0,0,1)$. 


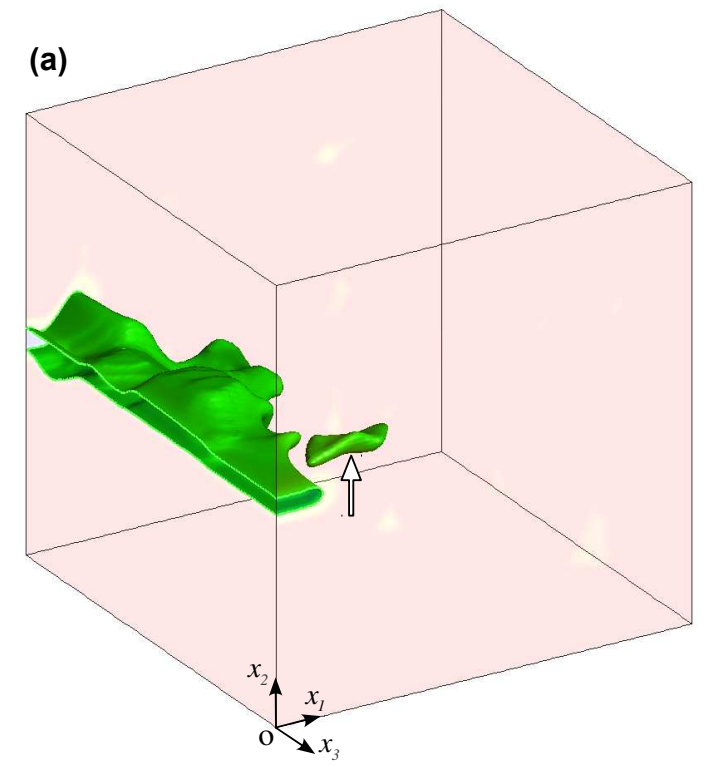

\section{(b)}

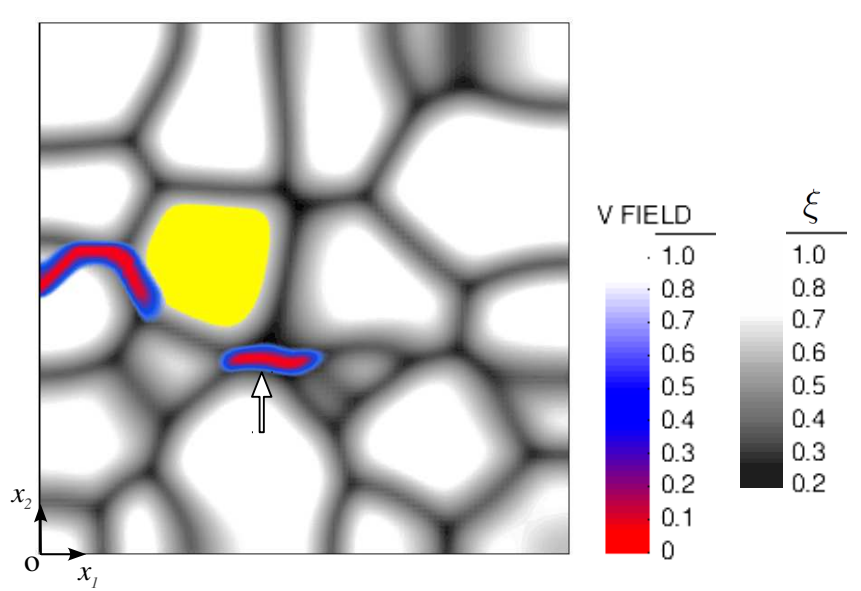

Figure A.8: Crack bridging in the fine-grain microstructure: (a) isosurface of the fracture parameter $v$ (b) distribution of $v$ (colour contour) and $\xi$ (gray contour), where grain boundaries and fracture zone are highlighted with the darker and red regions, respectively, and the area where $v>0.8$ is transparent to allow the visualization of the polycrystalline microstructure. The white arrow indicates the isolated crack from the main crack branch. The grain highlighted with yellow acts as a bridge between the main and isolated cracks. Figure (b) presents the contours in a cross section of the 3-D model which cuts the isolated crack with the surface unit normal $(0,0,1)$. This figure corresponds to the load step $\mathbf{c}$ and Fig. A.4(c). 

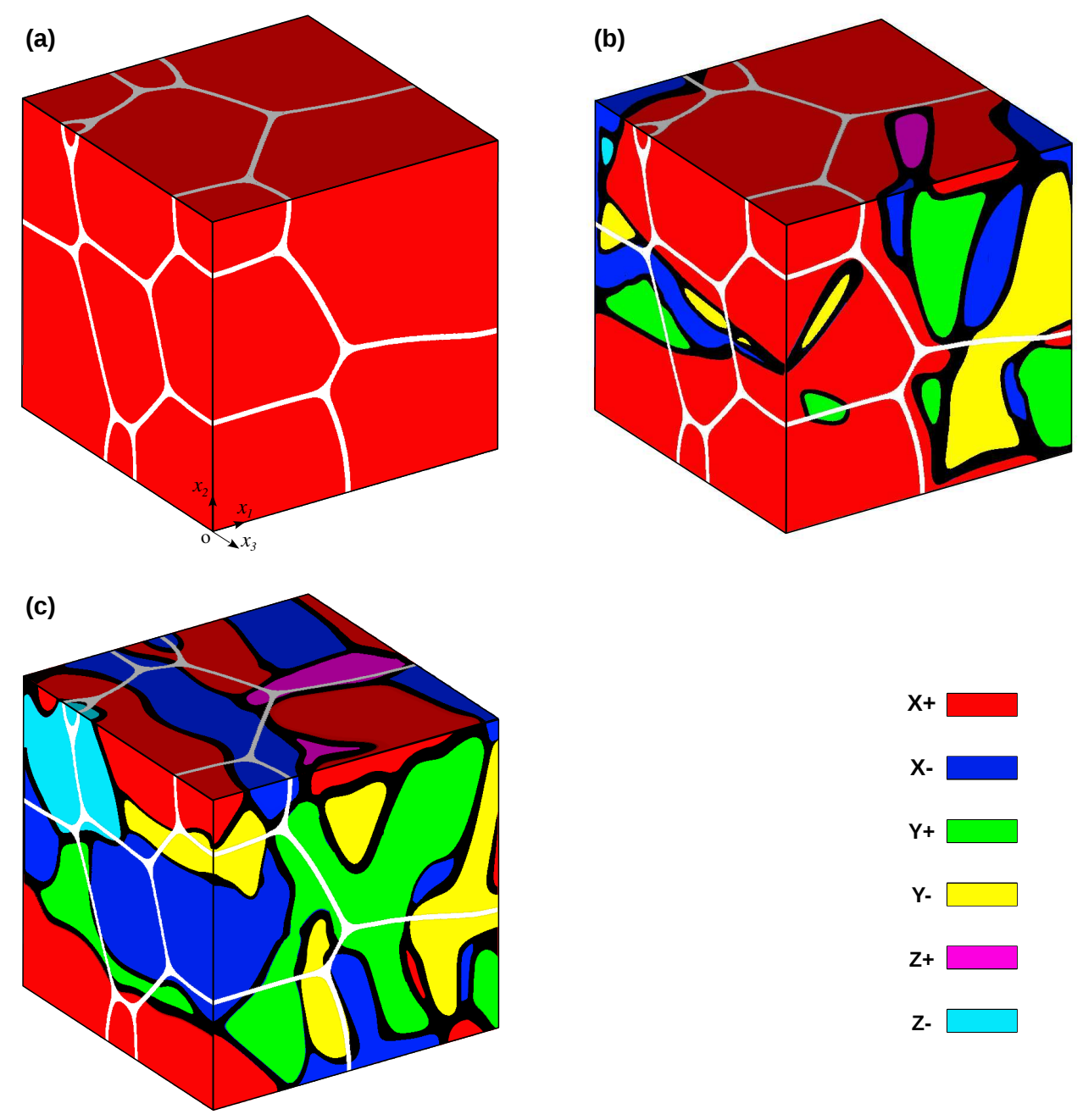

Figure A.9: Three snapshots of the evolution of the ferroelectric domain microstructure at load steps (a) $n=3$, (b) $n=100$, and (c) $n=190$. These figures correspond to the coarse-grain structure in Fig. A.2(b). Domain walls and grain boundaries are highlighted by black and white regions, respectively. There are six tetragonal variants, labeled as $\mathrm{X}+, \mathrm{X}-, \mathrm{Y}+, \mathrm{Y}-, \mathrm{Z}+$, and Z- with components of the normalized polarization in the local grain coordinate system $\left(p_{1}^{L L}>\tau\right),\left(p_{1}^{L L}<-\tau\right),\left(p_{2}^{\prime L}>\tau\right),\left(p_{2}^{\prime L}<-\tau\right),\left(p_{3}^{\prime L}>\tau\right)$, and $\left(p_{3}^{\prime L}<-\tau\right)$, respectively. To feature the domain wall regions, the value of $\tau$ is chosen as $20 \%$ of the normalized spontaneous polarization, i.e. $\tau=0.2$. The six tetragonal variants are colored according to the legend at bottom right. 


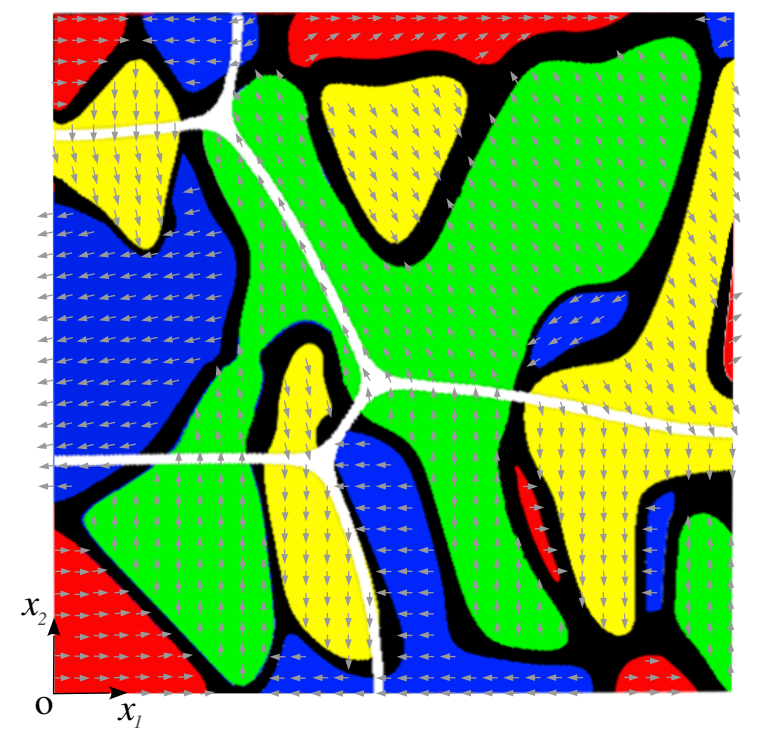

Figure A.10: Right view of Fig. A.9(c) where the gray arrows indicate the polarization in the global coordinate system. Domain walls and grain boundaries are represented by black and white regions, respectively. The tetragonal variants are colored with respect to the local coordinate system, according to the legend in Fig. A.9. The misalignment of the polarization vectors with respect to the global coordinate frame $\left(x_{2}-x_{1}\right)$ inside a single domain indicates the misalignment of the corresponding grains. For the sake of clarity, the polarization vectors are not depicted in the domain walls region. 
Table A.1: Normalized parameters

\begin{tabular}{cccccccc}
\hline & $\kappa_{0}^{\prime}$ & $\mu^{\prime}$ & $b_{1}^{\prime}$ & $b_{2}^{\prime}$ & $b_{3}^{\prime}$ & $a_{0}^{\prime}$ & \\
\cline { 2 - 6 } 133 & 36 & 1.4282 & -0.185 & 0.8066 & 0.2 & \\
\cline { 1 - 6 }$a_{1}^{\prime}$ & $a_{2}^{\prime}$ & $a_{3}^{\prime}$ & $a_{4}^{\prime}$ & $a_{5}^{\prime}$ & $\varepsilon_{0}^{\prime}$ & $G_{c}^{\prime}$ \\
\hline-0.007 & -0.009 & 0.018 & 0.0261 & 5 & 0.131 & 4 \\
\hline
\end{tabular}

